\title{
Development of a new washing machine in olive oil extraction plant: A first application of usability-based approach
}

\author{
Alessio Cappelli, ${ }^{1}$ Chiara Parretti, ${ }^{2}$ Enrico Cini, ${ }^{1}$ Paolo Citti ${ }^{2}$ \\ ${ }^{1}$ Department of Agriculture, Food, Environment and Forestry, University of Florence; ${ }^{2}$ Department of Innovation and \\ Information Engineering, Guglielmo Marconi University, Rome, Italy
}

\begin{abstract}
This paper suggests an approach based on usability engineering, used to assess the usability of machines and plants in the agrofood industry, aiming to enhance machines efficacy, efficiency and safety with a development focused on users' needs. Furthermore, the article examines the validation of the suggested approach through its application in a case study regarding a washing machine for the production of extra virgin olive oil. Firstly, it was necessary to identify the target cluster. For this reason, only Tuscan high quality extra virgin olive oil manufacturers were selected. The selected producers are of between 30 and 65 years old, with working experience in the field of at least 10 years. Once the cluster was identified, through brainstorming, the critical phases of the process were identified. According to the results, the washing process was considered the most critical phase. The reached conclusions were analysed and translated into technical aspects through the Quality Function Deployment. The predominant needs to be introduced in the new washing machine were related to the reduction of processing time, the improvement of washing system, the reduction of water consumption, and the increase of worker safety. Successively, a virtual prototype was developed and tested with 8 experts. Afterwards, a usability test was performed with 15 users. Two different olive washing machines were evaluated (traditional vs new machine). The Wilcoxon signed-rank test was utilised for users' scores interpre-
\end{abstract}

Correspondence: Alessio Cappelli, Department of Agriculture, Food, Environment and Forestry (DAGRI), University of Florence, Piazzale delle Cascine 15, Florence, Italy.

E-mail: alessio.cappelli@unifi.it

Key words: Agro-food industry; efficacy; human centred approach; machine design; safety; efficiency.

Acknowledgements: the authors would like to thank Mori-Tem and all the participants for their valuable contribution.

Received for publication: 20 February 2019.

Accepted for publication: 8 July 2019.

(C) Copyright: the Author(s), 2019

Licensee PAGEPress, Italy

Journal of Agricultural Engineering 2019; L:949

doi:10.4081/jae.2019.949

This article is distributed under the terms of the Creative Commons Attribution Noncommercial License (by-nc 4.0) which permits any noncommercial use, distribution, and reproduction in any medium, provided the original author(s) and source are credited. tation. According to $\mathrm{P}$-values (ranging from $\mathrm{P}<0.001$ to $\mathrm{P}=0.008$ ) several statistically significant differences emerged between the two washing machines. In particular, remarkable differences regarding efficacy, efficiency, usability, human-machine interaction, reliability and an increase in safety were highlighted. Moreover, the results indicate that the new washing machine is overall perceived as more usable machine. In conclusion, the results are encouraging and give robustness both to the proposed methodological approach and to the improvement of the new washing machine.

\section{Introduction}

Specific studies aimed to improve, in terms of reliability and productivity, the performance of the studied systems were achieved simultaneously to the economic and industrial development of various production sectors. The definition of efficiency, useful work/total work, allows us to understand the potential and the universal application of certain approaches. Moreover, the last twenty years have been characterised by the centrality of man in machine and processes planning (Longo et al., 2017). The development of products and processes designed with the purpose of improving working conditions through investment on human-machine interaction, should be interpreted as an enlightened way to increase the system's overall productivity, improve working conditions and reduce incurred costs (Zambon et al., 2018). Therefore, the suggested approach conceives usability, efficacy, safety, ergonomics and efficiency as essential elements in machines design and development. The term usability, described by ISO 9241 (Jokela et al., 2003), is defined as: effectiveness, efficiency and degree of satisfaction with which users reach certain goals in certain environments. The extension of the topics discussed in the 17 parts of ISO 9241 and their methodological approach, allows them to be applied outside the sector for which they have been elaborated and enables a diversified use of its content in various other categories of operators involved in the process of training, development and product control (Jokela et al., 2003).

In recent years, interest and scientific research have been focused on web usability, in particular on the usability assessment of software (Aykin, 2016), websites (García García et al., 2017) and apps (Balapour and Sabherwal, 2017). Lately, usability engineering, the discipline that studies techniques, methods and processes that can be used to design and develop usable systems, is coming back to the fore (Nielsen, 1994). A few studies which apply the usability engineering for the assessment of usability in machines, such as floor scrubbers (Sauer et al., 2010) and robotic sprayers for agriculture (Adamides et al., 2017), are reported in literature. In systems' design, the Human-centred approach (Longo et al., 2017) proved to be successful, because it deals with 
two important issues overlooked by other approaches, such as: i) the initial design may need to be changed for reasons not previously planned and controllable; ii) progress and new tools changes user's needs, generating new ones, which leads to the creation of new or optimised models.

The production of extra virgin olive oil (EVOO) is remarkably important in Europe, especially in Italy, with both economic and cultural relevance. The production of quality EVOO's is influenced by the optimal management of unit operations leading to its production, such as, harvesting, removing leaves and twigs, washing, olive milling, malaxing, centrifugation and filtration processes (Beghi et al., 2017). Certain steps of EVOO production, such as, post-harvest (Beghi et al., 2013), malaxing (Tamborrino et al., 2017), centrifugation (Boncinelli et al., 2009) and filtration (Costagli, 2018; Guerrini et al., 2015), were main topics of scientific research in the last few years, allowing a significant enhancement of the finished products' quality. The washing stage has been slightly neglected or leastwise, did not experience the same involvement, regarding experimental research efforts, despite representing one of the crucial steps for the quality of the finished product and for the reduction of the environmental impact. Few works on the treatment of olive washing wastewater have been carried out (Maza-Márquez et al., 2017), but studies aimed to improve the washing process, in terms of usability and safety, are missing, and therefore motivating this work.

The aim of this study is to apply the concepts of Usability Engineering to the sector of machine and plant for agro-food industry, through a comprehensive approach. With the intent of validating the proposed method, a case study pertaining olive washing machines is presented. The proposed method allows to develop usable machine and to assess the usability degree of machines and plants of agro-food industry, through specific usability tests. Furthermore, permit, through a dedicated technical data sheet, to communicate the degree of usability to other users.

\section{Materials and methods}

\section{Definition of the methodological approach for machines and manufacturing plants}

Usability is generally related to several aspects such as ease of use, ergonomics, efficacy, efficiency and reliability. These aspects are crucial elements in machines design and development. In fact, the proposed approach is built on four key elements able to ensure the best machine design with a development according to users' needs. These elements can be summarised as follow:

- Ergonomic aspect: Linked to human-machine interaction, which should be as easy as possible, in order to reduce costs, training time and errors, generating an improvement in workers' safety through an approach focused on users' activities, influencing all design phases. Moreover, this aspect concerns the evaluation of the introduced innovations; it contemplates whether the added functionalities enhances or decreases the machine's usability.

- Efficacy and efficiency aspect: this aspect concerns the effectiveness of the machine in goals achievement, considered in quantitative terms, and the efficiency, evaluated in qualitative terms, related to the reduction of costs/efforts in goals achievement.

- Reliability aspect: The improvement of machines and plants is strictly related to several aspects leading to a significant increase in reliability. Among these, user friendliness, the setting and use of machines and plants to their optimum operating conditions, minimizes errors and malfunctions, increasing the overall reliability.

- Usability and intuitiveness: Usable machines and plants are characterised by a significant reduction in time for training, with remarkable time saving and resources to fulfil it. These concepts, are also applicable to manuals; supporting the launch on the market of machines equipped of streamlined and easier to consult manuals. Furthermore, this aspect investigates the degree of usability perceived by the users during specific tests.

\section{Operative steps}

The presented methodological approach, in order to design and develop machines with high efficacy, efficiency and usability for agro-food industry, is based on the Deming approach (i.e. plan, do, check, act) (Johnson, 2002). As a matter of fact, it is possible to identify four operative steps listed as follows:

- Planning and programming Human-activity centred: Through questionnaires and interviews, all the information about the potential and evident needs, expectations, context, technical specifications, and, if possible, the willingness to pay are collected. The next step involves the processing and interpretation of the collected data in order to unfold the necessary requirements and performances according to production and economic objectives, also taking into consideration legal requirements related to safety, health and workers' training.

- Executive design: It concerns the realisation of practical design solutions, with a high degree of usability, through simulations, modelling and prototypes that enable the realisation of systems that satisfy user's requirements. Executive design involves the creation of a first prototype, using 3D simulation or 3D modelling, which can be presented to selected experts, in order to obtain an initial important feedback (Sauer et al., 2010). Furthermore, in this step, experts are essential, given the fact that they tend to discriminate more problems (Sauer et al., 2010). The realisation of several prototypes using 3D modelling, when needed, allows improving the reliability of the final prototype and finished products launched in the market, with considerable cost saving. According to the obtained results, it will be possible to continue with the executive design of the final prototype or editing it, depending on the first reactions of the consulted experts.

- Project control: This phase consists on verifying the satisfaction degree for the planned goals, and allows carrying out specific usability tests together with users. Depending on results of the final prototype usability test, a usability assessment data sheet will be compiled and attached to the product itself. This data sheet will support other consumers in the purchasing phase. The assessment data sheet allows to measure the level of usability and to easily communicate it to all other users, discriminating customers' needs orientation and the improved performance connected with greater usability compared to existing or competing models. Finally, if the obtained results are satisfying, the next and final stage of completion will be carried out. Otherwise, it will be necessary a re-editing of the final prototype or, if necessary, going back to the virtual prototype development step, as expected by the Deming approach (Johnson, 2002).

- Completion: This last step is based on demand quantification and on the structural design of the business organisation in an appropriate scale, in order to satisfy the product demand. This guarantees production, the complete availability of the product 
and it is proper commercialisation on the market. After the purchase phase, the user should not be abandoned, but instead, must join and feel part of a project oriented on his needs. Indeed, in addition to legal warranty, it is necessary and useful to keep in touch with consumers to obtain a feedback on usability. This important feedback, can be collected with questionnaires, submitted annually to customers, thanks to which it is possible to listen to users, collect their considerations and complaints in order to improve new models or for subsequent products.

\section{Expected results}

This approach allows gaining a series of multidisciplinary advantages, which can be summarised as follows:

Products with greater chances of success are built on their target consumers' needs and expectations. In fact, as highlighted in literature, customisation and ease of interaction are modern keys for the success of machine and products (Kwok and Wang, 2014). These are all relevant features in a market increasingly characterised by the reduction of time and the optimisation of production costs.

- Discovering overlooked potential uses behind the creation of new needs. The proposed approach allows developing the prototypes according to the specifications of experts and users. The realised machine should be able to convey its features to users who can report the usability degree and any missing or not inferred features through an assessment questionnaire at the end of the test. Nowadays, realising machines, plants and equipment's that are easy to interact with is essential, as reported in literature (Kwok and Wang, 2014).

- Increase reliability and workers 'safety. The realisation of several 3D prototypes, is related to an increase of reliability (Barnat et al., 2015). The reliability of a machine is also linked to its correct use; in this case, not only workers' training comes into play, but also the ease of use of the machine itself, that can only derive from a development focused on the optimisation of usability. This guarantees that users know the machine's functionalities and will use them at their optimal operating conditions, thus ensuring a significant increase in terms of reliability and safety (Zambon et al., 2018). The approach would allow an increase in machine reliability and safety through the optimisation of usability and human-machine interaction. This results in a purposeful use of a machine at its optimal operating conditions and in a specific safety training, but shorter and with clear information, unlike voluminous manuals, rarely consulted by workers. Finally, the proposed approach would reduce, in a significant way, time consumption and costs to train employees.

- Verify whether the information, communication and customer education are correctly perceived by users. Thanks to the information derived from tests with both experts and consumers, but also through questionnaires following the purchase. This is particularly relevant for manufacturing industry. Increasing brand trust. Brand trust is enhanced thanks to a production focused on satisfying consumer requirements, ensuring transparency and product safety (Lassoued and Hobbs, 2015). On this matter, a machine that is not satisfying users' needs can lead to a loss in the amount of customer. On the contrary, if a product is responsive to evident and potential consumer requirements, it will increase the brand's strength and trust, therefore increasing purchases and customer loyalty, simultaneously facilitating its penetration into new market sectors.

\section{Functioning principle and comparison between tradi- tional washing machine and new washing machine}

The olive washing process is currently still carried out with traditional washing machines. This process involves firstly the defoliation and deramification of olives, followed by their descent into the washing machine's water tank. In traditional washing machines, the washing process represents an important critical point (Di Giovacchino et al., 2002), because the water used to wash the olives should be completely drained and refilled at least every three cycles to be effective. Actually, this manufacturer's guideline is not followed word for word, leading to possible negative effects observable in the derived olive oils. Furthermore, this washing process is definitely ineffective because the dirty and contaminated water derived from the first wash is reused to clean the next batches, which instead of being cleaned get actually even more contaminated (Vichi et al., 2015). Some authors have suggested a different approach based on the continuous recycling of dirty water through a closed circuit washing phase in order to reduce both the microbial load and the environmental impact, but with unsatisfactory results (Vichi et al., 2015).

In addition, there are several other problems related to the usability of this traditional washing machine. Among these, the most significant ones are the remarkable waste of time during the operations connected with the complete removal, cleaning and filling of the water tank and the large consumption of water. Timing is crucial, especially during olive oil production period, characterised by intense non-stop work for a couple of months. In addition, the sustainability of food productions is nowadays a topic of crucial relevance; that's why the purpose of this paper is to apply the proposed approach for the development of an innovative washing machine, at maximum efficacy, efficiency, reliability, safety and usability, which allow to reduce/eliminate several of the detected problems.

The new washing machine, instead, is composed by a little stainless steel tray, dedicated to an initial rough wash, and a conveyor belt, which transfers the olives to the second part of the machine where two lines of washing nozzles fulfil the actual wash of the olives. This complete makeover allows to actually clean olives, instead of contaminating them again with dirty water, and, in addition, to limit water consumption. Moreover, towards the end, the drying process is carried out by three-air knife blowing systems with laminar flow. The approach of the new washing machine allows the completely removal of dirty water, enhancing the olives degree of cleanliness.

\section{Case study}

The methodological approach was implemented to the case study described in Figure 1. The figure shows the logical process used in this work for the usability evaluation. First, it was necessary to identify the target cluster. For this reason, only Tuscan high quality extra virgin olive oil manufacturers were selected. This choice of selecting only companies that produce high quality EVOO is due to a diversity of production processes, that involve the development of well identified and validated phases (Fregapane and Salvador, 2013), as shown in Figure 2.

The selected producers are of between 30 and 65 years old, with working experience, in the EVOO field, of at least 10 years. Once the cluster is identified through brainstorming, the critical phases of the process, able to influence the extra virgin olive oil quality, were identified. Three unit operations were specified: i) malaxing; ii) centrifugation with decanter; iii) washing process. According to the conclusions of the brainstorming, the washing 
process was considered the most critical phase; in fact, these results are in accordance with current literature showing the potential of olive microbial load, to influence the quality of the final oils (Cayuela et al., 2015) and the need to reduce the amount of added water to enhance the olive oil quality (Boncinelli et al., 2009). This highlights the need for innovations for the washing process, since the other two steps are already performed by advanced machines.

Following the brainstorming sessions related to the washing phase the reached conclusions were analysed and translated into technical aspects for the development of the product, through the quality function deployment (QFD) tool (Pacifici et al., 2017). The importance values, obtained by the average of the users' brainstorming scores, were multiplied by a correlation factor to obtain the values in the QFD boxes. The used correlation factors were one, three, and nine, according to the ability of the technical aspect to influence the voice of consumer (VOC) (one $=$ low importance, three $=$ medium importance, nine $=$ high importance). This was defined by the consulted experts. The weights were calculated by the sum of the values in columns. Moreover, the priority critical to quality (CTQ), were attributed in accordance with the obtained weights (from the highest to the lowest). Finally, the empty boxes indicates that the CTQ does not affect the VOC.

QFD was used to identify the main aspects which needed improvement/variations, compared to a traditional washing machine. In particular, the predominant needs to be introduced in the new washing machine were related to the reduction of processing time, the improvement of washing system, the reduction of water consumption, and the increase of worker safety. In order to validate the introduced aspects, a 3D model prototype (Figure 3b), developed according to the identified technical aspects, was tested by 8 sector experts. According to the results, few changes of the 3D prototype were introduced before the realisation of the new washing machine. Each of these ameliorations was evaluated in terms of veracity and correspondence with the needs stressed by users. This validation step with experts was advantageous as much for prototype reliability and usability evaluation as for the opportunity to attain an optimised prototype. Furthermore, 3D prototypes save time and money required for plentiful prototyping.

As a result, all these technical specifications were used for the development of the new washing machine (Figure 3b.1), tested with a group of 15 users who are acquainted with the extra virgin olive oil production. The participants were aged between 23 and 60 years old (average $=32.3 \pm$ standard deviation $=10.04$ ). The usability test was performed in the Montepaldi farm olive oil mill. The test concerned the usability assessment of two different washing machines illustrated in Figure 3; the first (a.1), not developed according to the proposed approach, is a traditional washing machine with features described in section 2; the second (b.1), on the contrary, is the new washing machine developed according to the usability based approach discussed in this paper.

Before performing the usability test with users, the test facilitator introduces users and explains the purposes of the test. Furthermore, a brief specific training for the understanding of both washing machines' operating principles, was performed. A single usability test with users was performed using the traditional washing machine and the new washing machine (Figure 3a.1 and b.1), using $100 \mathrm{~kg}$ of fresh olives for each test. The users had directly interacted with the machines and the facilitator, once explained the test and the tasks to fulfil, remained aside and intervened just in case of help requests. After training, users were asked to complete five tasks: i) turning on the washing machine and setting it to its optimum operating conditions; ii) fulfil the washing process following the olives and, if necessary, manually intervene; iii) carry out a selection of the outbound olives (removing the most damaged ones) and assessing the drying degree (where applicable); iv) perform all the necessary operations in order to clean the washing machine and to restore it to its optimal operating conditions; v) finish by turning the washing machine off.

Afterwards, the usability assessment was carried out through a post-study system usability questionnaire (PSSUQ) (Lewis, 1995). The original questionnaire, made of 19 items, was slightly readapted to the case study by replacing two irrelevant questions (i.e. 16 and 17) with two others regarding safety, in line with the suggested approach. Moreover, the term system was replaced by washing machine, with the intent of enhancing user understanding. In Table 1 is the adjusted PSSUQ employed in this paper, pairing each question and its assessed usability aspect. The participants evaluated each item on a 7-point Likert scale (from strongly

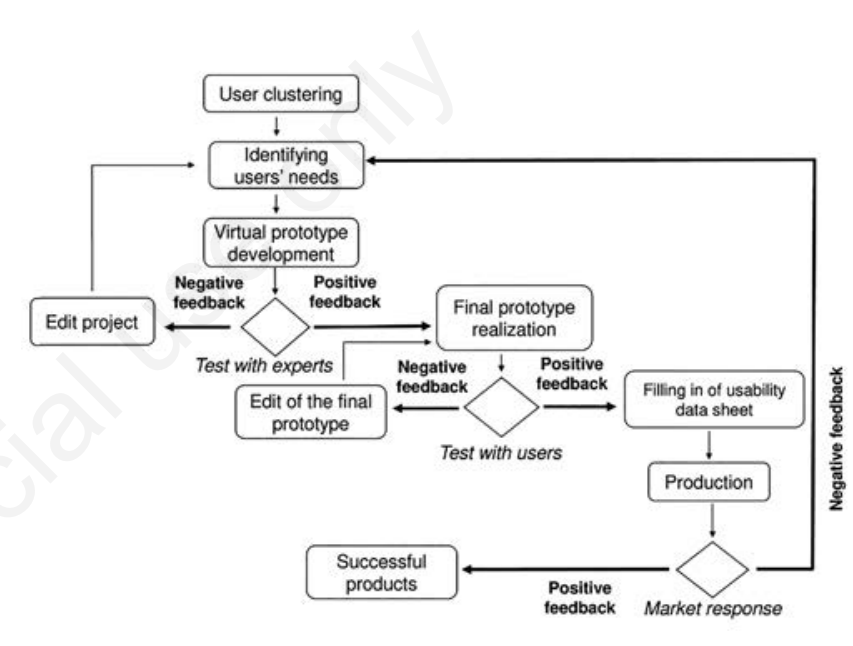

Figure 1. Logical process for the development of the described activity, with identified key decisions points.

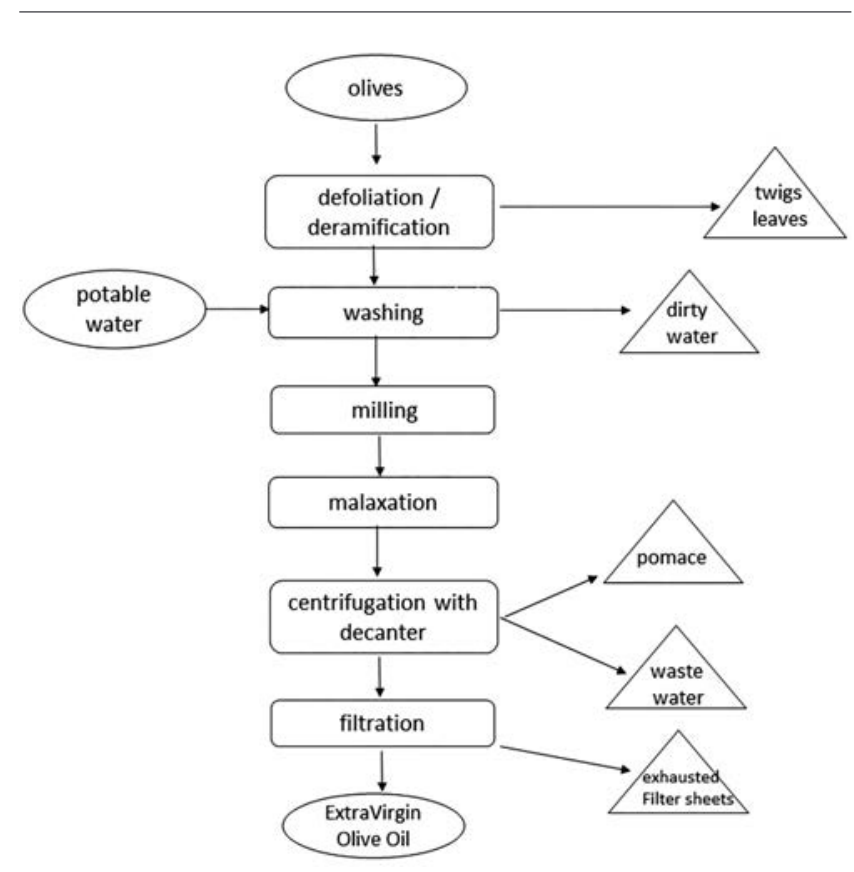

Figure 2. Flow sheet of high quality extra virgin olive oil production. 
disagree to strongly agree). The results of the PSSUQ were analysed using non-parametric statistical test. In particular, the purpose was of evaluating usability differences between the two different olive washing machines shown in Figure 3, (i.e. a. $1=$ not developed according to the proposed approach, b.1 = developed according to the usability based approach). The Wilcoxon signedrank test was chosen to assess statistical differences between the scaled scores assigned by the same 15 users (i.e. paired condition) (Montgomery, 2017). The Wilcoxon signed-rank test was carried out using the statistical software R.

\section{Results and discussion}

\section{Experimental results}

Results pertaining needs' identification, first prototyping and testing with experts

As reported in section 2.5, the QFD tool was used to identify main aspects requiring improvement/variations, transforming

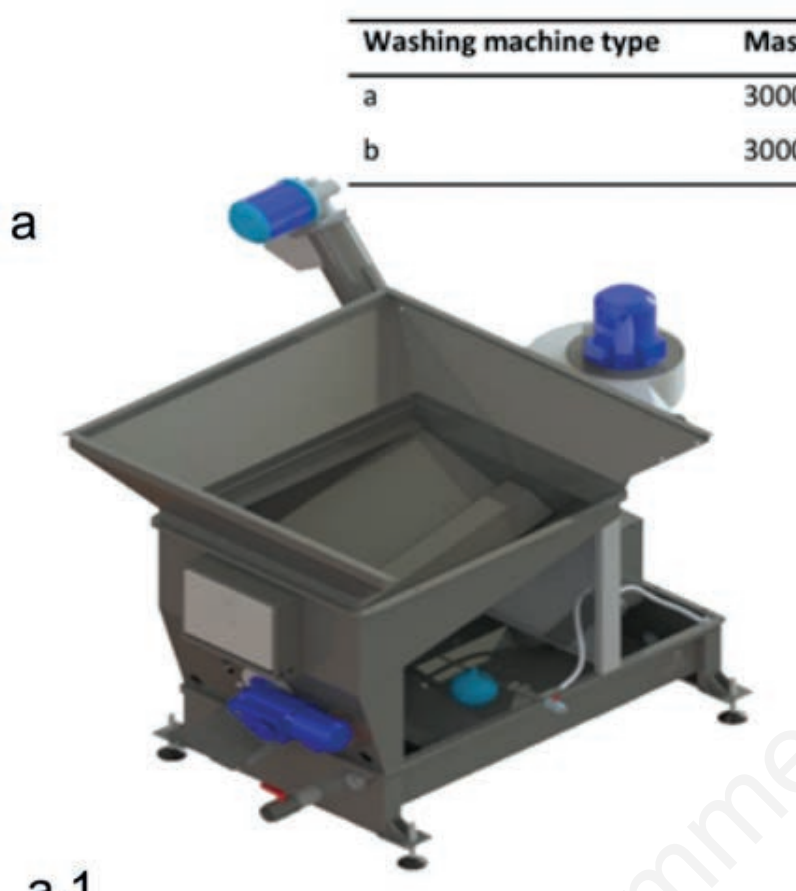

a.1
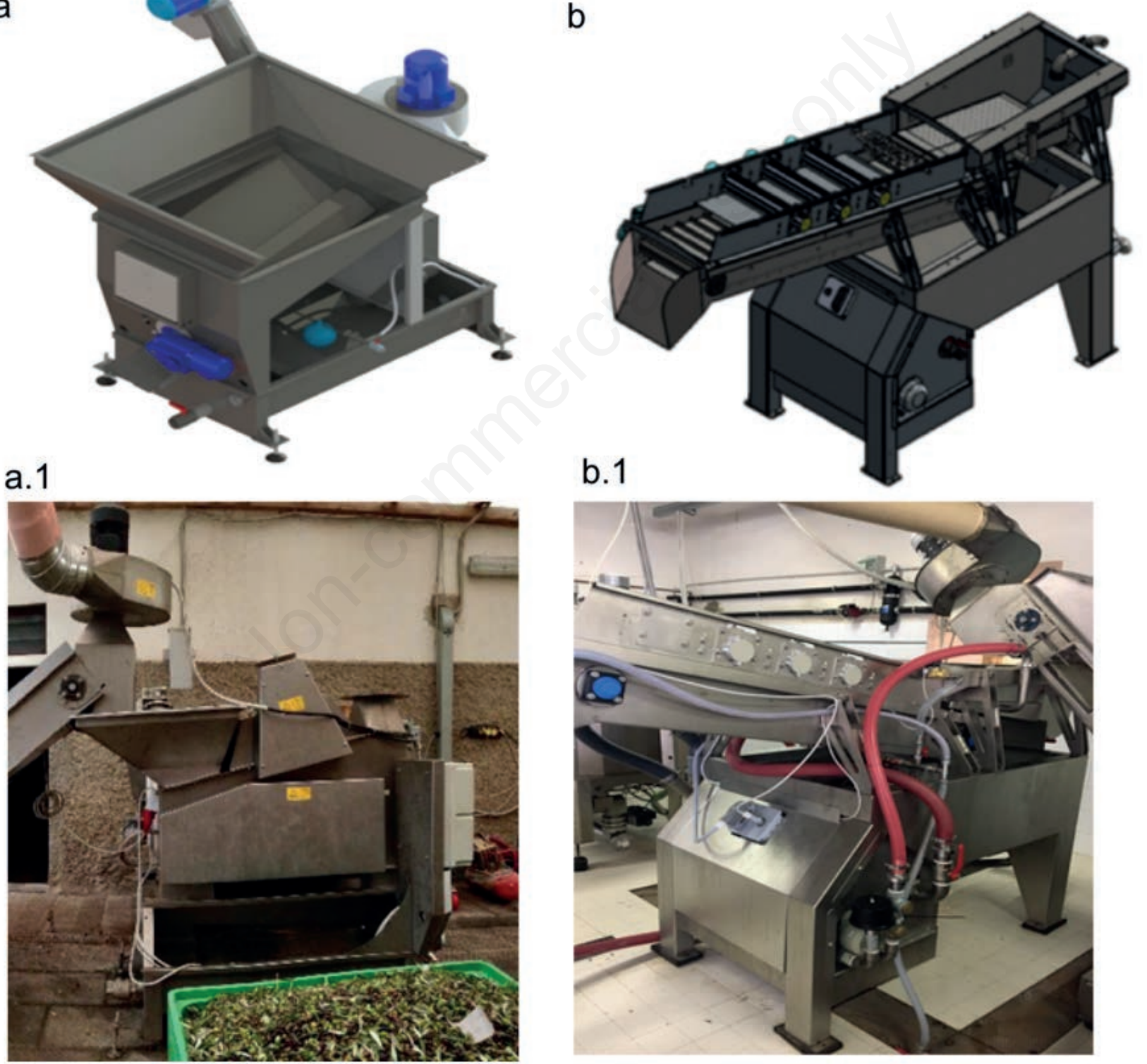

Figure 3. Washing machines tested in this study: a) traditional washing machine prototype and a.1) traditional washing machine not optimised in usability term. b) virtual prototype of the new washing machine and b.1) new washing machine developed according to the usability-based approach. 
users' indications into technical features CTQ. Results of QFD are displayed in Table 2. As expected, the users of the chosen cluster have highlighted several critical points related to the ineffective washing system, to the need for speed and a better cleaning system and, finally, the need to reduce water consumption; as a matter of fact, these aspects have assumed the highest priority degree in the QFD. According to these three significant critical matters, it was necessary to dispose the washing tank and a complete makeover of the washing and machine cleaning systems. For this reason, the new washing machine is made of a little stainless steel tray, dedicated to an initial rough wash of the olives and then by a conveyor belt, which transfers the olives to the second part of the machine. Afterwards, through two lines of washing nozzles, the actual wash of the olives is fulfilled, followed, towards the end, by the drying

Table 1. Adjusted version of the post-study system usability questionnaire and match with the assessed aspects.

\begin{tabular}{lll} 
Questionnaire items & Aspect of the proposed approach \\
1. & Overall, I am satisfied with how easy it is to use this washing machine & Usability and intuitiveness \\
2. & It was simple to use this washing machine & Usability and intuitiveness \\
\hline 3. & I could effectively complete the tasks and scenarios using this washing machine & Efficacy and efficiency aspect \\
4. & I was able to complete the tasks and scenarios quickly using this washing machine & Efficacy and efficiency aspect \\
\hline 5. & I was able to efficiently complete the tasks and scenarios using this washing machine & Efficacy and efficiency aspect \\
6. & I felt comfortable using this washing machine & Ergonomic aspect \\
\hline 7. & It was easy to learn how to use this washing machine & Usability and intuitiveness \\
\hline 8. & I believe I could become productive quickly using this washing machine & Efficacy and efficiency aspect \\
\hline 9. & The washing machine did not froze or cause any interruptions during the test phase & Reliability aspect \\
10. & Whenever I made a mistake using the washing machine, I could recover easily and quickly. & Reliability aspect \\
\hline 11. & The information given by the control panel and by the washing machine was clear. & Ergonomic aspect \\
12. & It was easy to find the information I needed. & Ergonomic aspect \\
\hline 13. & The information provided for the washing machine was easy to understand & Reliability aspect \\
14. & The information was effective in helping me complete tasks and scenarios. & Efficacy and efficiency aspect \\
\hline 15. & The organisation of information for setting the washing machine to the optimal operating conditions was clear & Reliability aspect \\
16. & I consider the automation level of the washing machine as optimal & Ergonomic aspect \\
\hline 17. & I consider the interaction with the washing machine during a manual intervention as safe & Ergonomic aspect \\
18. & This washing machine has all the functions and capabilities I expect it to have. & Ergonomic aspect \\
\hline 19. & Overall, I'm satisfied with this washing machine & Usability and intuitiveness \\
\hline
\end{tabular}

Table 2. Output of quality function deployment which summarises the main aspects needing improvement/variations into the washing machine. The importance values were obtained by the average of the users' brainstorming scores. The weights were calculated by the sum of the values in columns. The priority critical to quality (CTQ)'s, were attributed in accordance with the obtained weights (from the highest to the lowest). The empty boxes indicates that the CTQ does not affect the voice of consumer.

\begin{tabular}{|c|c|c|c|c|c|c|c|c|}
\hline \multirow[t]{2}{*}{ Voice of consumer } & \multirow[b]{2}{*}{ Importance } & \multicolumn{6}{|c|}{ Critical to quality } & \multirow[b]{2}{*}{$\begin{array}{l}\text { Olives } \\
\text { selection } \\
\text { system }\end{array}$} \\
\hline & & $\begin{array}{l}\text { Improvement } \\
\text { of machine } \\
\text { cleaning system }\end{array}$ & $\begin{array}{l}\text { Improvement } \\
\text { of the washing } \\
\text { n system }\end{array}$ & $\begin{array}{l}\text { Used } \\
\text { materials }\end{array}$ & $\begin{array}{l}\text { Drying of } \\
\text { outbound } \\
\text { olives }\end{array}$ & $\begin{array}{c}\text { Automation } \\
\text { level }\end{array}$ & $\begin{array}{l}\text { Washing } \\
\text { tank }\end{array}$ & \\
\hline $\begin{array}{l}\text { Reduction of } \\
\text { processing time }\end{array}$ & 6.8 & 61.2 & 61.2 & 20.4 & & 61.2 & 61.2 & \\
\hline $\begin{array}{l}\text { Washing } \\
\text { improvement } \\
\text { (reduction of } \\
\text { microbial load) }\end{array}$ & 6.3 & 56.7 & 56.7 & 56.7 & 56.7 & & 56.7 & 6.3 \\
\hline $\begin{array}{l}\text { Ease of machine } \\
\text { cleaning }\end{array}$ & 5.6 & 50.4 & 50.4 & 50.4 & & 16.8 & 50.4 & \\
\hline Olives drying & 6.3 & & & & 56.7 & & & \\
\hline Worker safety & 6.7 & 60.3 & 20.1 & 60.3 & & 60.3 & 60.3 & 6.7 \\
\hline $\begin{array}{l}\text { Reduction of water } \\
\text { consumption }\end{array}$ & 6.7 & 60.3 & 60.3 & & & & 60.3 & \\
\hline $\begin{array}{l}\text { Chance to select the olives } \\
\text { before milling process }\end{array}$ & 6.3 & & & & & & & 56.7 \\
\hline Weights & & 288.9 & 248.7 & 187.8 & 113.4 & 138.3 & 288.9 & 69.7 \\
\hline Priority critical to quality & & $1 \mathrm{~b}$ & 2 & 3 & 5 & 4 & la & 6 \\
\hline
\end{tabular}


process carried out through three air knife blowing systems with laminar flow. This new washing approach allows to actually clean olives, instead of contaminating them again with dirty water (as occur with traditional washing machines). Furthermore, according to the consumptions measured during trials, the new washing machine allows to limit water consumption from $210 \mathrm{~L} / \mathrm{h}$ to 150 $\mathrm{L} / \mathrm{h}$, as shown in Figure 3.

Furthermore, the improvement of the machine cleaning system is strictly related to the materials used and to the automation level that are immediately following CTQs. Instead, the cleaning system of the traditional washing machine (i.e. Figure 3a.1) requires, first of all, an interruption of production, then a treatment using sodium hydroxide (i.e. caustic soda) with subsequent rinsing out and a treatment with citric acid. After this, another rinse out is required. Finally, is necessary to refill the water tank; process which requires approximately $15-20 \mathrm{~min}$ alone. This procedure is carried out by the olive oil mill operator, with a significant exposure to chemical risk and a remarkable waste of time. By contrast, the new cleaning system comprises a single and rapid cleaning phase (which requires a few minutes) with $60^{\circ} \mathrm{C}$ water supplied by a pressure washer, granting a significant reduction of time and an increasing safety level for workers. Furthermore, the dirty water and the other

Table 3. Mean scores obtained in the post-study system usability questionnaire for the two washing machines (i.e. Figure 3a.1 and 3b.1) and the P-values assessed utilising the Wilcoxon signed-rank test.

\begin{tabular}{|c|c|c|c|}
\hline $\begin{array}{l}\text { Questionnaire } \\
\text { item }\end{array}$ & $\begin{array}{l}\text { Average scores for } \\
\text { traditional washing } \\
\text { machine (Figure 3a.1) }\end{array}$ & $\begin{array}{c}\text { Average scores for } \\
\text { the new washing } \\
\text { machine (Figure } 3 \mathrm{~b} .1 \text { ) }\end{array}$ & P-values \\
\hline Question n.1 & $4.83 \pm 1.26$ & $6.3 \pm 0.41$ & $\mathrm{P}=0.001$ \\
\hline Question n.2 & $5.13 \pm 1.17$ & $6.03 \pm 0.55$ & $\mathrm{P}=0.007$ \\
\hline Question n.3 & $3.3 \pm 1.47$ & $6.5 \pm 0.5$ & $\mathrm{P}<0.001$ \\
\hline Question n.4 & $2.93 \pm 1.50$ & $6.43 \pm 0.53$ & $\mathrm{P}=0.001$ \\
\hline Question n.5 & $2.93 \pm 1.45$ & $6.47 \pm 0.48$ & $\mathrm{P}<0.001$ \\
\hline Question n.6 & $3.97 \pm 1.17$ & $6.33 \pm 0.59$ & $\mathrm{P}<0.001$ \\
\hline Question n.7 & $5.63 \pm 0.72$ & $6.3 \pm 0.49$ & $\mathrm{P}=0.001$ \\
\hline Question n.8 & $2.43 \pm 1.08$ & $6.5 \pm 0.5$ & $\mathrm{P}<0.001$ \\
\hline Question n.9 & $4.17 \pm 1.22$ & $6.23 \pm 0.59$ & $\mathrm{P}=0.001$ \\
\hline Question n.10 & $3.4 \pm 1.71$ & $6.43 \pm 0.62$ & $\mathrm{P}=0.002$ \\
\hline Question n.11 & $5.03 \pm 1.39$ & $6.5 \pm 0.46$ & $\mathrm{P}=0.001$ \\
\hline Question n.12 & $4.73 \pm 1.50$ & $6.3 \pm 0.49$ & $\mathrm{P}=0.004$ \\
\hline Question n.13 & $5.73 \pm 1.07$ & $6.47 \pm 0.52$ & $\mathrm{P}=0.008$ \\
\hline Question n.14 & $3.9 \pm 1.48$ & $6.67 \pm 0.41$ & $\mathrm{P}<0.001$ \\
\hline Question n.15 & $3.8 \pm 1.67$ & $6.4 \pm 0.60$ & $\mathrm{P}=0.001$ \\
\hline Question n.16 & $3.47 \pm 1.84$ & $6.3 \pm 0.56$ & $\mathrm{P}=0.001$ \\
\hline Question n.17 & $3.7 \pm 1.37$ & $6.63 \pm 0.44$ & $\mathrm{P}<0.001$ \\
\hline Question n.18 & $2.9 \pm 1.39$ & $6.2 \pm 0.56$ & $\mathrm{P}<0.001$ \\
\hline Question n.19 & $3.9 \pm 1.36$ & $6.4 \pm 0.43$ & $\mathrm{P}<0.001$ \\
\hline
\end{tabular}

\section{USABILITY DATA SHEET}

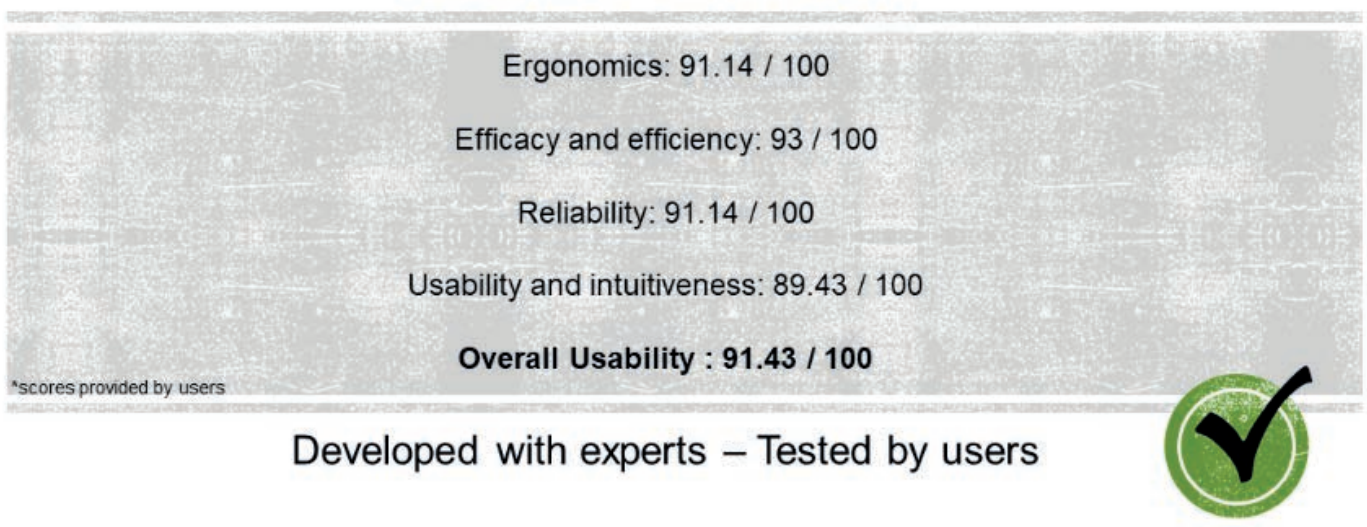

Figure 4. Usability data sheet of the new washing machine developed according the scores provided by users. 
residues are carried onto the underlying tank and are immediately expelled by the wastewater management system, saving a remarkable amount of time. The decrease of time spent to clean the machine and the elimination of chemical risk exposure in the washing machine developed according to the proposed approach, are related to the use of easily washable materials and to the presence of the underlying tank which allows to immediately drain waste water and other residues preventing encrustations and other related issues, which can only be removed after chemical treatments.

Finally, the last two technical features identified by the selected users for improving the washing machine were the drying of the outbound olives and the possibility to select the olives before the milling process. The first, is strictly related to the improvement of the olives' washing process, since only the removal of the entire washing water droplets ensures a significant reduction of microbial load; in addition, it ameliorates the quality of both the process itself (i.e. less water is introduced in the EVOO production plant) and of the product (i.e. the reduction of the microbial load guarantees safer products, without sensory defects). The latter is, instead, connected to the simplification of the machine cleaning process and to the EVOO quality improvement, since the removal of most of the damaged olives allows not to process contaminated or poor quality drupes.

According to the results of the QFD, the virtual prototype development was accomplished. The application of the proposed approach allowed the identification of several critical issues, which led to a complete reinvention of the olives washing system. This prototype was tested by 8 sector experts who gave a positive feedback provided that an extension of the latest part of the conveyor belt will be added to the new washing machine, in order to facilitate the selection of olives. Moreover, they suggested to leave in plain sight the two lines of washing nozzles in order to enhance inspection and maintenance, and to add a third air knife blower to dry olives, so to improve the drying process itself. In agreement with experts' suggestions, the final version of the virtual prototype was achieved (Figure $3 b$ ). The final version of the virtual prototype was utilised for the realisation of the new washing machine. Figure $3 \mathrm{~b} .1$, shows the new washing machine utilised in usability tests with users.

\section{Usability test results}

The results of usability tests carried out with users are specified in Table 3. Table 3 details the average scores obtained in the PSSUQ for the two washing machines tested in this study (i.e. Figure 3a.1 and 3b.1), and the P-values resulted from the Wilcoxon signed-rank test. According to P-values, several statistically significant differences emerged between the two washing machines. In particular, remarkable differences regarding efficacy, efficiency, usability, human-machine interaction, reliability and an increase in safety were highlighted. These results are encouraging and give robustness both to the four main aspects that are the essence of the proposed methodological approach and to the improvement of the newly developed washing machine. Furthermore, these results indicate that the new washing machine is overall perceived by users as a more usable machine, as confirmed by the high P-value of question number nineteen.

Finally, according to the average of the obtained scores for each of the four aspects, it was possible to realise the data sheet, which will support other consumers in the purchasing phase. Figure 4 shows the data sheet that will be paired to the new washing machine.

\section{Conclusions}

This work highlights how usability, a key element in the new technology sector, can be used in a conscious and profound way, as a tool for improvement in the efficacy and efficiency of machine and plants for food production. Regarding the new washing machine developed according to the proposed approach, the double validation with both experts and users added countless benefits. The first validation with experts, allowed to detect a greater number of problems related to usability and to improve technical features minimising prototyping. The second validation with users, permitted to assess the actually perceived usability of the washing machines. Furthermore, the development of the new washing machine allowed to deal and solve all the critical issues detected by the selected users, bringing innovative solutions, which led to the gain in usability and safety for the new washing machine. Moreover, it is important to underline that the advantages of this double validation are reflected both on the new washing machine and on the methodological approach. Returning to the washing machine improvements, a significant reduction of water consumption (i.e. 60 litters per hour) and of the processing time (predominantly related to the machine cleaning) were verifiable in this new washing machine. Finally, all the innovations concerning the olives drying and the possibility to select the olives before milling, did not complicate the machine; on the contrary, they increased its perceived usability degree.

Concerning the developed approach, it allowed to obtain reliable and representative results. Considering its great versatility, this approach might be applicable to other productive sectors, obviously, through a careful reconsideration of the four main aspects on which the approach is based on. Furthermore, the obtained results, show that the four main elements which shape the approach proposed in this paper, are effective and efficient in understanding and assessing the machine's usability. In conclusion, the modified PSSUQ performed accurately, however, the design of a specific questionnaire able to assess the usability of machines and plants in the agro-food industry, remains interesting. This will be examined in future studies.

\section{References}

Adamides G., Katsanos C., Parmet Y., Christou G., Xenos M., Hadzilacos T., Edan Y. 2017. HRI usability evaluation of interaction modes for a teleoperated agricultural robotic sprayer. Appl. Ergon. 62:237-46.

Aykin N. (Ed.). 2016. Usability and internationalisation of information technology. CRC Press, Boca Raton, FL, USA.

Balapour A., Sabherwal R. 2017. Usability of apps and websites: a meta-regression study. Twenty-third Americas Conference on Information Systems, Boston, USA. Available from: https://aisel.aisnet.org/cgi/viewcontent.cgi?article=1301\&context=amcis 2017

Barnat S., Guédon-Gracia A., Frémont H. 2015. Virtual prototyping in a design-for-reliability approach. Microelectr. Reliab. 55:1849-54.

Beghi R., Giovenzana V., Civelli R., Cini E., Guidetti R. 2013. Characterisation of olive fruit for the milling process by using visible/near infrared spectroscopy. J. Agric. Engine. 44:e8.

Beghi R., Giovenzana V., Guidetti R., Cappelli A., D’Antoni A., Menditto N., Cini E. 2017. Exploitation of technological innovations along the olive oil milling process for an optimisation 
of the plant performance. pp 285-288 in International AIIA Conference, Università degli studi di Bari Aldo Moro, Bari, Italy.

Boncinelli P., Daou M., Cini E., Catalano P. 2009. A simplified model for designing and regulating centrifugal decanters for olive oil production. Trans. ASABE 52:1961-8.

Cayuela J.A., Gómez-Coca R.B., Moreda W., Pérez-Camino M.C. 2015. Sensory defects of virgin olive oil from a microbiological perspective. Trends Food Sci. Technol. 43:227-35.

Costagli G. 2018. The use of disc stack centrifuge in the virgin olive oil industry. J. Agric. Engine. 49:75-80.

Di Giovacchino L., Sestili S., Di Vincenzo D. 2002. Influence of olive processing on virgin olive oil quality. Eur. Lipid Sci. Technol. 104:587-601.

Fregapane G., Salvador M.D. 2013. Production of superior quality extra virgin olive oil modulating the content and profile of its minor components. Food Res. Int. 54:1907-14.

García García M., Carrillo-Durán M.V., Tato Jimenez J.L. 2017. Online corporate communications: website usability and content. J. Commun. Manage. 21:140-54.

Guerrini L., Masella P., Migliorini M., Cherubini C., Parenti A. 2015. Addition of a steel pre-filter to improve plate filter-press performance in olive oil filtration. J. Food Engine. 157:84-87.

Johnson C.N. 2002. The benefits of PDCA. Quality Progr. 35:120.

Jokela T., Iivari N., Matero J., Karukka M. 2003. The standard of user-centered design and the standard definition of usability: analysing ISO 13407 against ISO 9241-11. pp 53-60 in Proceedings of the Latin American conference on Humancomputer interaction (ACM).

Kwok T.H., Wang C.C. 2014. Shape optimisation for human-centric products with standardised components. Comput. Aid. Design 52:40-50.

Lassoued R., Hobbs J.E. 2015. Consumer confidence in credence attributes: the role of brand trust. Food Policy 52:99-107.
Lewis J.R. 1995. IBM computer usability satisfaction questionnaires: psychometric evaluation and instructions for use. Int. J. Hum. Comput. Interact. 7:57-78.

Longo F., Nicoletti L., Padovano A. 2017. Smart operators in industry 4.0: a human-centered approach to enhance operators' capabilities and competencies within the new smart factory context. Comput. Industr. Engine. 113:144-59.

Maza-Márquez P., González-Martínez A., Rodelas B., GonzálezLópez J. 2017. Full-scale photobioreactor for biotreatment of olive washing water: structure and diversity of the microalgaebacteria consortium. Bioresour. Technol. 238:389-98.

Montgomery D.C. 2017. Design and analysis of experiments. John Wiley and Sons, Philadelphia, PA, USA.

Nielsen J. 1994. Usability engineering. Elsevier, Amsterdam, The Netherlands.

Pacifici B., Parretti C., Girgenti A., Citti P. 2017. Axiomatic design for an efficient development of optimised RPM systems. In MATEC Web of Conferences, EDP Sciences, 127:01010.

Sauer J., Seibel K., Rüttinger B. 2010. The influence of user expertise and prototype fidelity in usability tests. Appl. Ergon. 41:130-40.

Tamborrino A., Squeo G., Leone A., Paradiso V.M., Romaniello R., Summo C., Caponio F. 2017. Industrial trials on coadjuvants in olive oil extraction process: effect on rheological properties, energy consumption, oil yield and olive oil characteristics. J. Food Engine. 205:34-46.

Vichi S., Boynuegri P., Caixach J., Romero A. 2015. Quality losses in virgin olive oil due to washing and short $\square$ term storage before olive milling. Eur. J. Lipid Sci. Technol. 117:12.

Zambon I., Piergentili A., Salvati L., Monarca D., MatyjasŁysakowska P., Colantoni A. 2018. Applied research for a safer future: exploring recent job accidents in agriculture, Italy (2012-2017). Processes 6:87. 